Archiv für Gewerbepathologie und Gewerbehygiene, Bd. 13, S. 616-618 (1955).

From the Institute of Industrial Health (Director: Dr. O. T. MaLLerY, JR.) and the School of Public Health (Dean: Dr. H. F. VAUGHaN)

University of Michigan, Ann Arbor.

\title{
Protection of Workers against Dermatitis on Exposure to Hydrazine Hydrobromide Soldering Flux*.
}

By

Warren A. Cook, Ann Arbor.

(Eingegangen am 10. Februar 1955.)

Soldering flux has long been a cause of dermatitis where this material has spattered on the skin as the hot iron or torch is applied to the fluxed metal. The principal fluxes causing such dermatitis have been hydrochloric acid and stannic chloride, the action being that of a primary irritant.

Both of these substances tend to corrode the metal parts on which they are used and research has been undertaken to find a flux which would act on the metal surface in such a manner as to permit the solder to adher but which would avoid the undesired corrosive action. Hydrazine hydrobromide and also the hydrochloride possess these attributes and already, according to TROYAN, they are being used for this purpose on a wide scale. Brass and copper are the metals with which this flux is chiefly used, but flux compositions are also being developed for use with aluminum.

The hydrazine monohydrobromide does not dissociate in water (AUDRIETH and OGG) and accordingly can be used in aqueous solution, $2 \%$ being a usual concentration. The method of application varies widely with the materials being soldered, the extent of mass production of them and the ingenuity of the individual plant production engineer. In some plants the solution is applied automatically by means of a rotary brush; in others the solution is sprayed on the parts as they pass through an enclosure on a conveyor; in still others, the simple procedure of applying the flux solution by means of a hand brush is used. In the two former methods, exclosures are provided with exhaust ventilation as it was learned early in the introduction of this material that dermatitis occurred whenever the solution spattered on the skin of workers in the vicinity of the operation.

After the hydrazine hydrobromide has been applied to the metal parts, soldering is done usually with torch or hot iron using soft solder in the form of wire or rod. Occasionally the parts may be dipped in a bath of molten solder.

* Dedicated to the 80th birthday of Professor Heinrich Zanganr. 
When the elevated temperature of the soldering operation contacts the hydrazine hydrobromide, pyrolysis occurs causing dissociation of the compound with the production of a white fume. Although we do not have complete analyses of the products of pyrolysis, the substances disseminated on application of heat include some spatter of unchanged hydrazine hydrobromide, possibly some vaporized unchanged salt, nitrogen, ammonia, probably ammonium bromide and hydrogen bromide, and free hydrazine vapor. Air analyses conducted by a colleague, J. H. Fanney, JR., have shown presence of hydrazine and of bromide ion on pyrolysis of hydrazine hydrobromide from the $2 \%$ solution under conditions closely simulating those of soldering.

It has long been known that hydrazine vapor is highly irritating to the nose and throat (CURTIUs). A naval technical committee publication in 1948 is cited by Cомsтоск et al. as observing that exposure to hydrazine vapor produces irritation to the nose and throat, followed by itching, burning and swelling of the eyes. Dermatitis on skin contact has been reported in the manufacturer's literature (CLARK). In one plant where the fume from the flux rose past the faces of the workers conducting the soldering operation, dermatitis of the face occurred, Exhaust ventilation was installed to prevent this contact and the dermatitis ceased.

After the soldering has been completed, the soldered parts and the clamps and frames holding the parts together must be handled. It was found that there was sufficient residue of the flux on the surface of these pieces to cause dermatitis on the hands of all who came in contact with them. Workers who clamped together parts to be soldered developed dermatitis even before any flux was applied to the parts. It was noted that residual flux on the clamps provided the contact. A simple expedient of washing the clamps before reuse was put into practice and cases of dermatitis among these workers no longer occurred.

Protection of workers handling parts with surfaces containing hydrazine hydrobromide consisted largely of strict adherence to wearing of rubber gloves, rubber or plastic aprons and arm coverings. Dermatitis of the hand continued after rubber gloves were being worn. It was noted that contact with hydrazine hydrobromide occured when the second glove was removed. The workers were then required to wash their gloves before removal. If gloves for any reason were to be removed before being washed, the first glove was to be partly stripped from the hand, the second glove removed and then the first glove completely stripped without touching its outside contaminated surface.

One organization with much opportunity for contact found that vaseline (petroleum jelly) was effective in preventing skin irritation and that lanolin was somewhat less effective. 
In four plants concerning which we are specifically informed, dermatitis occurred whenever there was contact with the hydrazine hydrobromide. In fact in our industrial hygiene laboratory, a student working with the problem of hydrazine hydrobromide pyrolytic products developed a mild dermatosis. All of these cases cleared on avoiding contact with the flux.

In concluding this discussion of the production of many cases of dermatitis on use of hydrazine hydrobromide, together with methods observed for protection of workers in prevention of such dermatitis, it should be pointed out that hydrazine, one of the demonstrated products of pyrolysis of this flux, is an exceedingly toxic substance as discussed by Krop in a review of the toxicology of hydrazine. ComsTock et al. found that $18 \mathrm{mg}$. per cubic meter ( 15 parts per million) produced marked effects in dogs and eventually some deaths. A third of this concentration was found to produce very little pathology in dogs over six months of exposure and it is suggested by these investigators that the maximum acceptable concentration (MAC) is below $6 \mathrm{mg}$. per cubic meter $(5 \mathrm{ppm})$. No reports of systemic effects resulting from use of hydrazine hydrobromide or the hydrochloride as a flux have come to our attention. Operations which we have observed have not appeared to involve sufficient exposure to cause such effect.

However, where there may be some confinement of the fume from this flux or where the nature of the work may cause the fume to pass the breathing zone of the worker, it would be wise to be on the alert for anorexia and weight loss which Comstock et al. suggest may be the earliest symptoms and may constitute a warning sign of impending health hazard. Certainly if there is any question of excessive exposure, air analyses should be made and the results correlated with presence or absence of symptoms in man. In event of symptomatology referable to hydrazine, the exposure of the entire group of workers should be reduced appreciably below that of the affected worker in addition to providing treatment for him as suggested by KRoP.

\section{Literature.}

Audrieth, L. F., and B. A. OgG: Chemistry of hydrazine. New York: John Wiley \& Sons 1951. - Clark, C. C.: Hydrazine. Baltimore: Mathieson Chemical Company 1953. - Comstock et al.: Inhalation toxicity of hydrazine vapor. A.M.A. Arch. Industr. Hyg. a .Occup. Med. 10, 476 (1954). — Cun'rus, T.: UUber Diamid (Hydrazin). Ber. dtsch. chem. Ges. 20, 1633 (1887). - Krop, S. : Toxicology of hydrazine - - a review. A.M.A. Arch. Industr. Hyg. a. Occup. Med. 9, 199 (1954).Troyan, J. E.: Properties, production, and uses of hydrazine. Ind. Engng. Chem. 45, 2608 (1953).

Prof. Warken A. Cook, School of Public Health, 109 So. Observatory St., Ann Arbor, Michigan, U.S.A. 